Lara, N.C.C.

INSTITUTO DE ESTUDOS DE SAÚDE SUPLEMENTAR

\title{
DESPESAS COM INTERNAÇÕES DE OPERADORA DE AUTOGESTÃO SEGUNDO O PORTE HOSPITALAR
}

\section{$>$ INTRODUÇÃO}

O estudo analisa as despesas com internação segundo o porte hospitalar. A referência bibliográfica internacional, sobre porte de hospitais relata que para um hospital ter economia de escala é necessário ter entre 200 a 400 leitos. O Brasil depende grandemente de hospitais: eles respondem por $70 \%$ dos atendimentos de emergência, 27\% do atendimento ambulatorial e por quase todas as internações.

\section{$>$ METODOLOGIA}

O estudo analisa o dispêndio assistencial de uma operadora de autogestão do Estado de São Paulo por porte de hospital privado no período de 2015, são 383 hospitais privados no Brasil, 98,8\% estão localizados no Estado de São Paulo.

\section{$>$ RESULTADOS}

Os hospitais de grande porte são os que apresentaram maior número de internações e um maior gasto médio por internação sendo 5.013 e R\$30.729, respectivamente. Seguido dos hospitais de médio (4.284 e $R \$ 24.406)$ e pequeno porte (1.158 e $\mathrm{R} \$$ 11.641). Na análise de comparação entre gasto médio da operadora com internação por CID mais recorrentes segundo o porte hospitalar em 2015 verificou que a maioria das doenças os custos são mais elevados nos hospitais de grande porte, exceto para os CIDs de "Infecção do trato urinário de localização não especificada" que o maior valor foi nos hospitais de médio porte ( $R \$ 56.393 .18$ ) e "Dor abdominal e pélvica" que foi de $R \$ 24.783,01$, também em hospitais de médio porte.

Tabela 1. Distribuição das despesas de internações segundo o tamanho do porte do hospital, 2015.

\begin{tabular}{|c|c|c|c|}
\hline $\begin{array}{c}\text { PORTE } \\
\text { HOSPITALAR }\end{array}$ & $\begin{array}{l}\text { DESPESAS DE } \\
\text { INTERNAÇÃo }\end{array}$ & $\begin{array}{l}\text { QUANTIDADE } \\
\text { DE NÚMERO DE } \\
\text { INTERNAÇÕES }\end{array}$ & $\begin{array}{c}\text { GASTO } \\
\text { MÉDIO POR } \\
\text { PROCEDIMENTO } \\
\text { (R\$) }\end{array}$ \\
\hline PEQUENO PORTE & $\mathrm{R} \$ 13.480 .702,41$ & 1.158 & 11.641 \\
\hline MÉDIO PORTE & $R \$ 104.553 .889,28$ & 4.284 & 24.406 \\
\hline GRANDE PORTE & R\$ 154.046.422,44 & 5.013 & 30.729 \\
\hline Total & $\mathrm{R} \$ 272.081 .014,13$ & 10.455 & $R \$ 26.024,01$ \\
\hline
\end{tabular}

Tabela 2. Gastos de internação dos hospitais segundo o procedimento, 2015.

\begin{tabular}{|c|c|c|c|c|}
\hline \multicolumn{5}{|c|}{ HOSPITAL PEQUENO PORTE } \\
\hline $\mathrm{N}^{\circ}$ & PROCEDIMENTOS POR CID & VALORES & $\begin{array}{l}\text { QUANTIDADE DE } \\
\text { PROCEDIMENTOS }\end{array}$ & GASTO MÉDIO \\
\hline 1 & INFECÇÃO DO TRATO URINÁRIO DE LOCALIZAÇÃO NÃO ESPECIFICADA & $\mathrm{R} \$ 866.070,57$ & 8 & $R \$ 108.258,82$ \\
\hline 2 & PNEUMONIA P/MICROORG NE & $\mathrm{R} \$ 853.909,43$ & 14 & $\mathrm{R} \$ 60.993,53$ \\
\hline 3 & INSUFICIÊNCIA CARDÍACA CONGESTIVA & $\mathrm{R} \$ 686.964,54$ & 10 & $R \$ 68.696,45$ \\
\hline 4 & PNEUMONIA NÃO ESPECIFICADA & $R \$ 685.150,34$ & 25 & $\mathrm{R} \$ 27.406,01$ \\
\hline 5 & ANEMIA EM NEOPLASIAS (CO0-D48+) & $\mathrm{R} \$ 681.906,78$ & 1 & $R \$ 681.906,78$ \\
\hline 6 & SÍNCOPE E COLAPSO & $R \$ 590.106,36$ & 5 & $\mathrm{R} \$ 118.021,27$ \\
\hline 7 & OUTRAS PNEUMONIAS DEVIDAS A MICROORGANISMOS NÃO ESPECIFICADOS & $\mathrm{R} \$ 441.463,76$ & 2 & $R \$ 220.731,88$ \\
\hline 8 & ACIDENTE VASCULAR CEREBRAL, NÃO ESPECIFICADO COMO HEMORRÁGICO OU ISQUÊMICO & $\mathrm{R} \$ 430.441,32$ & 11 & $\mathrm{R} \$ 39.131,03$ \\
\hline 9 & OUTRAS PNEUMONIAS BACTERIANAS & $\mathrm{R} \$ 393.087,48$ & 8 & $\mathrm{R} \$ 49.135,94$ \\
\hline 10 & HIPOSMOLARIDADE E HIPONATREMIA & $R \$ 385.026,12$ & 2 & $\mathrm{R} \$ 192.513,06$ \\
\hline \multicolumn{5}{|c|}{ HOSPITAL MÉDIO PORTE } \\
\hline 1 & INFECÇÃO DO TRATO URINÁRIO DE LOCALIZAÇÃO NÃO ESPECIFICADA & $R \$ 3.778 .342,87$ & 67 & $R \$ 56.393,18$ \\
\hline 2 & SEPTICEMIA NÃO ESPECIFICADA & $R \$ 2.914 .354,38$ & 37 & $R \$ 78.766,33$ \\
\hline 3 & BRONCOPNEUMONIA NÃO ESPECIFICADA & $R \$ 2.865 .078,30$ & 85 & $\mathrm{R} \$ 33.706,80$ \\
\hline 4 & ACIDENTE VASCULAR CEREBRAL, NÃO ESPECIFICADO COMO HEMORRÁGICO OU ISQUÊMICO & $R \$ 2.613 .252,35$ & 65 & $\mathrm{R} \$ 40.203,88$ \\
\hline 5 & PNEUMONIA NÃO ESPECIFICADA & $R \$ 2.249 .954,54$ & 63 & $\mathrm{R} \$ 35.713,56$ \\
\hline 6 & DISPNÉIA & $R \$ 2.248 .870,20$ & 17 & $R \$ 132.286,48$ \\
\hline 7 & PNEUMONIA P/MICROORG NE & $R \$ 1.995 .650,68$ & 54 & $\mathrm{R} \$ 36.956,49$ \\
\hline 8 & EMBOLIA E TROMBOSE DE OUTRAS VEIAS ESPECIFICADAS & $\mathrm{R} \$ 1.937 .079,72$ & 4 & $R \$ 484.269,93$ \\
\hline 9 & OUTRAS PNEUMONIAS BACTERIANAS & $R \$ 1.886 .398,16$ & 7 & $R \$ 269.485,45$ \\
\hline 10 & INSUFICIÊNCIA CARDÍACA CONGESTIVA & $R \$ 1.625 .853,75$ & 35 & $\mathrm{R} \$ 46.452,96$ \\
\hline \multicolumn{5}{|c|}{ HOSPITAL GRANDE PORTE } \\
\hline 1 & ENCEFALITE PELO VÍRUS DO HERPES ZOSTER (G05.1*) & $\mathrm{R} \$ 1.647 .201,70$ & 1 & $R \$ 1.647 .201,70$ \\
\hline 2 & OCLUSÃO E ESTENOSE DA ARTÉRIA VERTEBRAL & $R \$ 627.023,81$ & 1 & $R \$ 627.023,81$ \\
\hline 3 & COLANGITE & $R \$ 2.691 .533,84$ & 6 & $\mathrm{R} \$ 448.588,97$ \\
\hline 4 & COMA NÃO ESPECIFICADO & $\mathrm{R} \$ 433.312,08$ & 1 & $R \$ 433.312,08$ \\
\hline 5 & TRAUMATISMO NÃO ESPECIFICADO & $\mathrm{R} \$ 856.136,84$ & 2 & $\mathrm{R} \$ 428.068,42$ \\
\hline 6 & OUTR DOENC VASCULARES PERIFERICAS & $R \$ 403.095,68$ & 1 & $R \$ 403.095,68$ \\
\hline 7 & CIRROSE HEPÁTICA ALCOÓLICA & $R \$ 711.351,72$ & 2 & $R \$ 355.675,86$ \\
\hline 8 & OUTR TRAUM DA CABECA E OS NE & $\mathrm{R} \$ 674.891,25$ & 2 & $R \$ 337.445,63$ \\
\hline 9 & OROFARINGE, NÃO ESPECIFICADA & $\mathrm{R} \$ 334.377,00$ & 1 & $R \$ 334.377,00$ \\
\hline 10 & OUTR AFECCOES PELE TEC SUBCUTANEO DOENC COP & $R \$ 325.537,05$ & 1 & $R \$ 325.537,05$ \\
\hline
\end{tabular}

Tabela 3. Comparação entre gasto médio da operadora com internação por CID recorrentes (em ordem decrescente) segundo porte hospitalar, 2015.

\begin{tabular}{|c|c|c|}
\hline PROCEDIMENTOS POR CID & MÉDIO & GRANDE \\
\hline $\begin{array}{l}\text { ACIDENTE VASCULAR CEREBRAL, } \\
\text { NÂO ESECCIFICADO COMO } \\
\text { HEMORRÁGICO OU ISQUÊMICO }\end{array}$ & $\mathrm{R} \$ 40.203,88$ & $R \$ 65.262,58$ \\
\hline PNEUMONIA NÃO ESPECIFICADA & $\mathrm{R} \$ 35.713,56$ & $R \$ 63.980,89$ \\
\hline $\begin{array}{c}\text { BRONCOPNEUMONIA NÃO } \\
\text { ESPECIFICADA }\end{array}$ & $\mathrm{R} \$ 33.706,80$ & $\mathrm{R} \$ 52.682,13$ \\
\hline $\begin{array}{l}\text { INSUFICIÊNCIA CARDÍ́ACA } \\
\text { CONGESTIVA }\end{array}$ & $6.452,96$ & 477,15 \\
\hline $\begin{array}{l}\text { INFECÇÃO DO TRATO URINÁRIO DE } \\
\text { LOCALIZAÇÃO NÃO ESPECIFICADA }\end{array}$ & $R \$ 56.393,18$ & $\mathrm{R} \$ 39.433,40$ \\
\hline INSUF CARDIACA & $\mathrm{R} \$ 27.195,10$ & $\mathrm{R} \$ 29.316,41$ \\
\hline NEOPL MALIG DA MAMA & $R \$ 19.178,90$ & $R \$ 25.535,42$ \\
\hline $\begin{array}{l}\text { DIARRÉIA E GASTROENTERITE DE } \\
\text { ORIGEM INFECCIOSA PRESUMÍVEL }\end{array}$ & $R \$ 10.856,58$ & $R \$ 21.947,67$ \\
\hline DOR ABDOMINAL E PELVICA & $\mathrm{R} \$ 24.783,01$ & $R \$ 20.212,08$ \\
\hline DENGUE [DENGUE CLÁSSICO] & $\mathrm{R} \$ 3.367,61$ & $R \$ 4.954,67$ \\
\hline
\end{tabular}

\section{CONCLUSÃO}

Segundo Forgia (2009), estas grandes diferenças podem ocorrer devido a práticas clinicas adotadas por cada hospital e a diferença de perfil de cada paciente entre os hospitais, outras causas podem estar atreladas a natureza do hospital, se é uma entidade sem fins lucrativos ou não, assim como, a diferença de tempo médio de internação, associadas à severidade de casos individuais ou à eficiência na utilização dos recursos a falta de padronização em metodologias de custeio; e sistemas deficientes de informação de pacientes. Portanto, o estudo conclui que existe uma necessidade de mudar a estrutura do sistema, para um atendimento em que visa prevenir doenças, do que ter o foco em tratar as doenças; e dos hospitais obterem um processo mais eficiente e eficaz, padronizando os processos e práticas clínicas no atendimento ao paciente. www.iess.org.br

(11) 3706-9747 - contato@iess.org.br
Rua Joaquim Floriano, 1052 - Conj. 42 Itaim Bibi - Paulo/SP - 04534-004 